\title{
Intensified Surveillance AFter Surgery for Colorectal Cancer SigNIFICANTLY IMPROVES SURVIVAL
}

\author{
T. Laubert, F. G. Bader, E. Oevermann, T. Jungbluth, L. Unger, U. J. Roblick, H.-P. Bruch, L. Mirow \\ Department of Surgery, University of Schleswig-Holstein, Campus Lübeck, Lübeck, Germany
}

\begin{abstract}
Background: Postoperative surveillance after curative resection for colorectal cancer has been demostrated to improve survival. It remains unknown however, whether intensified surveillance provides a significant benefit regarding outcome and survival. This study was aimed at comparing different surveillance strategies regarding their effect on long-term outcome.

Methods: Between 1990 and 2006, all curative resections for colorectal cancer were selected from our prospective colorectal cancer database. All patients were offered to follow our institution's surveillance programm according to the ASCO guidelines. We defined surveillance as "intensive" in cases where $>70 \%$ appointments were attended and the program was completed. As "minimal" we defined surveillance with $<70 \%$ of the appointments attended and an incomplete program. As "none" we defined the group which did not take part in any surveillance.

Results: Out of 1469 patients 858 patients underwent "intensive", 297 "minimal" and 314 "none" surveillance. The three groups were well balanced regarding biographical data and tumor characteristics. The 5 -year survival rates were $79 \%$ (intensive), 76\% (minimal) and $54 \%$ (none) (OR 1.480, (95\% CI 1.135-1.929); $p<0.0001)$, respectively. The 10 -year survival rates were $65 \%$ (intensive), $50 \%$ (minimal) and 31\% (none) $(p<0.0001)$, respectively. With a median follow-up of 70 months the median time of survival was 191 months (intensive), 116 months (minimal) and 66 months (none) $(p<0.0001)$. After recurrence, the 5 -year survival rates were $32 \%$ (intensive, $p=0.034$ ), 13\% (minimal, $p=0.001$ ) and $19 \%$ (none, $p=0.614$ ). The median time of survival after recurrence was 31 months (intensive, $p<0.0001), 21$ months (minimal, $p<0.0001$ ) and 16 month (none, $p<0.0001$ ) respectively.

Conclusion: Intensive surveillance after curative resection of colorectal cancer improves survival. In cases of recurrent disease, intensive surveillance has a positive impact on patients' prognosis. Large randomized, multicenter trials are needed to substantiate these results.
\end{abstract}

Key-words: Cancer, colorectal, surveillance

\section{INTRODUCTION}

Colorectal cancer (CRC) constitutes the third most common cause of cancer death in the US [1]. In Europe, there are more than 350000 new cases each year
[2]. Despite advances in treatment regimes, at least $40 \%$ of patients with UICC stage II or III disease will experience local or distant recurrence [3]. The purpose of surveillance following curative therapy of colorectal cancer is early identification of recurrent disease which can potentially be cured by further surgical interventions.

The assumption that early detection of recurrent disease leads to better outcome has been fortified by several studies [4-7]. Meta-analyses showed a modest but significant survival benefit for patients monitored by an intensive surveillance program after resection of CRC [8-11]. Additional surgery can cure some patients who have limited local recurrence or distant metastases, thus emphasising the importance of surveillance after colorectal cancer surgery.

The guidelines for surveillance are a matter of constant debate. In 2005, the American Society of Clinical Oncology (ASCO) revised the 2000 guidelines for surveillance based on recent studies and reviews $[12,13]$. Nevertheless, it remains controversial which diagnostic tests should be performed at what point of a surveillance program, underlining the need for further studies.

Recently, we published the results of a prospective analysis for patients undergoing a surveillance program following surgical resection for CRC between 1979 and 1990 [14, 15]. We were now able to analyze almost 1600 patients who received curative surgical therapy throughout the period between 1990 and 2006 with regard to differences in prognosis correlated to the intensity of the surveillance program. The purpose of this prospective analysis was to evaluate whether an intensive surveillance program is beneficial for patients after resection for CRC.

\section{Material And Methods}

Between January 1990 and December 2006 we performed 2079 resections on patients with CRC at the Department of Surgery, University of Schleswig-Holstein, Campus Lübeck, Germany. All data concerning patients undergoing a colorectal resection at our institution are prospectively collected in a "colorectal cancer" database. We performed surveillance according to ASCO guidelines $[12,13]$. At the time of discharge all patients were informed about the necessity for a regular and complete postoperative surveillance. Surveillance data of individual patients was recorded in a 
standardized questionnaire for the first ten years after resection. Thereafter, the responsible physician was contacted and the questionnaire was sent to each patient on a yearly basis. If there was any missing data, we addressed the registration office for residents for support.

We defined the surveillance as "intensive" in cases where the participation was regular $(>70 \%$ of the given appointments were attended) and the program was completed. As "minimal" we defined surveillance with less than $70 \%$ of the appointments attended and an incomplete program. An incomplete program was defined as a divergence between tests scheduled according to the ASCO-guidelines and the testes actually obtained. As "none" we defined cases which did not undergo surveillance at all. Consequently, we categorised groups of patients as "intensive", "minimal" and "none" surveillance. Patients presenting with rectal and colon cancer simultaneously were defined as rectal cancer patients. In this study, we compared the three different groups with regard to local and distant recurrence, the rate of curative resection after recurrence and the accordant 5- and 10-year survival rates. Recurrence was defined as the occurrence of a local (intra and/or extramural) or distant tumor after curative resection (R0). Only curative resections (R0) were identified from our database. Patients with R1 or R2 resection as well as patients with synchronous, not resectable distant metastases were excluded. Further exclusion criteria were local excisions, inability to cooperate, ulcerative colitis- and Crohn's disease associated cancer and hereditary cancer syndromes (FAP, HNPCC).

\section{STATISTICAl ANALYsis}

Continuous variables were expressed as mean \pm standard deviation. The length of surveillance and followup was expressed as median and range. Statistical analysis was carried out univariately using the $\chi^{2}$-Test and results were considered significant with $\mathrm{p}<0.05$. A $\mathrm{p}$-value lower/less than 0.0001 was not calculated exactly and is noted as $\mathrm{p}<0.0001$. Probabilities of overall survival rate were calculated according to the KaplanMeier method. A Log-Rank-test was applied for comparison with $\mathrm{p}<0.05$ considered statistically significant. Starting time for survival analysis was the day of surgical resection of the primary colorectal cancer. Estimations of the relative risk were expressed as odds ratio (OR) with a 95\% confidence interval (95\% CI). All calculations were performed by using the SPSS 14 (SPSS Inc, Chicago, IL, USA) software.

\section{RESULTS}

Between 1990 and 2006, a total of 2079 patients underwent surgery for CRC. Subtracting the cases with synchronous, not resectable distant metastases as well as R1 and R2 resections, 1567 patients were operated with curative intent.

The in-hospital mortality rate was 4.4\% $(\mathrm{n}=69)$ with a 30 -day mortality rate of $3.9 \%(n=61)$. Another 29 patients $(1.9 \%)$ died within the first three months after surgery without any cause related to surgery or tumor. This resulted in a total of 1469 patients who were potentially available for surveillance and thus constituted the study population. Within this group $793(54 \%)$ primary tumors were located in the colon, and $676(46.0 \%)$ in the rectum including $20(1.4 \%)$ synchronous carcinomas.

A total of 263 patients $(17.9 \%)$ received a complete regime of adjuvant chemo- or radiotherapy including $128 / 793(16.1 \%)$ patients with a carcinoma of the colon and 135/676 (20\%) with a carcinoma of the rectum. With $65.0 \%$ the main reasons not to complete or begin an adjuvant therapy were refusal by the patients and a decline in general condition. Distribution among the groups with intensive, minimal and none was equal.

Out of 1469 patients, the majority underwent intensive surveillance $(\mathrm{n}=858 ; 58.4 \%), 297$ patients (20.2\%) underwent a minimal program (overall participation $78.6 \%$ ). The group of patients not participating in surveillance accounted for 314 patients (21.4\%). The groups of intensive, minimal and none surveillance were well balanced regarding stage of disease and gender (Table 1). Notably, the cohort with none surveillance was older and in a worse general condition. No patient was lost to follow-up.

Table 1. Patients' clinical and biographical data.

\begin{tabular}{llll}
\hline $\begin{array}{l}\text { Follow-up [median months] } \\
\text { Study population }\end{array}$ & & $\begin{array}{c}70 \text { (range } 1-211) \\
\mathrm{n}=1469\end{array}$ \\
\hline Surveillance & $\begin{array}{l}\text { intensive } \\
\mathrm{n}=858\end{array}$ & $\begin{array}{l}\text { minimal } \\
\mathrm{n}=297\end{array}$ & $\begin{array}{l}\text { none } \\
\mathrm{n}=314\end{array}$ \\
\hline Gender & & & \\
male & $478(56 \%)$ & $150(51 \%)$ & $151(48 \%)$ \\
female & $380(44 \%)$ & $147(49 \%)$ & $163(52 \%)$ \\
Age [years] & $64,5( \pm 10.9)$ & $67,3( \pm 11.5)$ & $73,7( \pm 11.6)$ \\
UICC-stage & & & \\
Stage I & $293(34 \%)$ & $104(35 \%)$ & $102(33 \%)$ \\
Stage II & $266(31 \%)$ & $95(32 \%)$ & $116(37 \%)$ \\
Stage III & $273(32 \%)$ & $92(31 \%)$ & $92(29 \%)$ \\
Stage IV & $26(3 \%)$ & $6(2 \%)$ & $4(1 \%)$ \\
\hline
\end{tabular}

\section{SURVIVAL}

The 5-year survival rates for the different groups were $79 \%$ (intensive), 76\% (minimal) and 54\% (none), respectively. The inter-group comparison revealed a statistically significant improved overall survival for the intensive surveillance group (Table 2; intensive vs. minimal $\mathrm{p}<0.0001$, intensive vs. none $\mathrm{p}<0.0001$, mini-

Table 2. Survival rates depending on the intensity of surveillance.

\begin{tabular}{llll}
\hline Surveillance & 5-year survival & 10-year survival & p-value \\
\hline intensive & $79 \%$ & $65 \%$ & $<0.0001$ \\
minimal & $76 \%$ & $50 \%$ & $<0.0001$ \\
none & $54 \%$ & $31 \%$ & $<0.0001$ \\
\hline
\end{tabular}




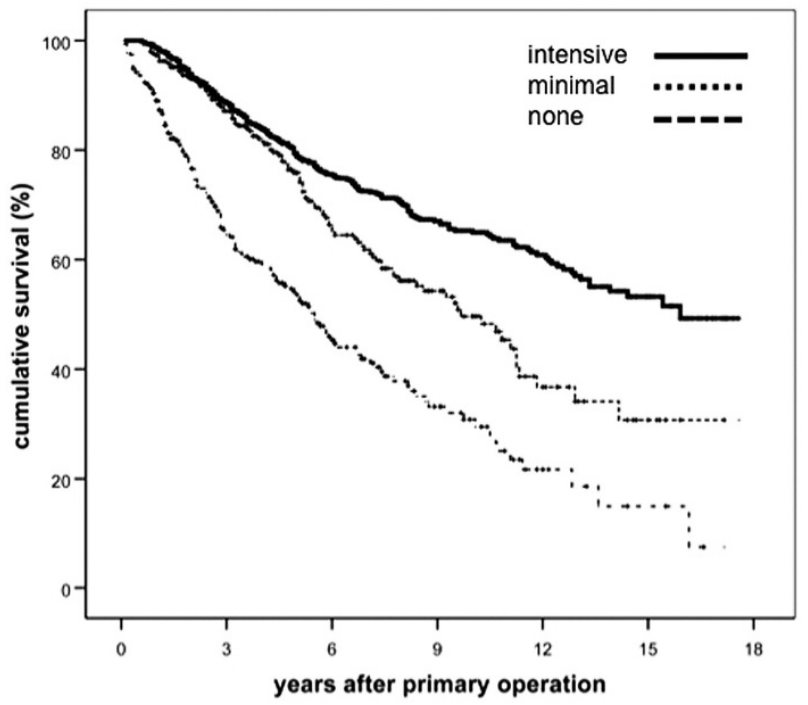

Fig. 1. Survival after curative resection of colorectal cancer depending on the intensity of surveillance.

mal vs. none $\mathrm{p}<0.0001)$. The 10 -year survival rate for the group with intensive surveillance was $65 \%, 50 \%$ for the group with minimal surveillance and 31\% for the group with none surveillance (intensive vs. minimal $p<0.0001$, intensive vs. none $p<0.0001$, minimal vs. none $p<0.0001$; Fig. 1).

After a median follow-up of 70 months the calculated median time of survival was 191 months (intensive), 116 months (minimal) and 66 months (none), respectively. The differences in the inter-group comparison were statistically significant (intensive vs. minimal $p<0.0001$, intensive vs. none $p<0.0001$, minimal vs. none $p<0.0001$ )

The odds ratio for a 5-year survival comparing intensive and minimal surveillance was 1.480 (95\% CI 1.135-1.929). The comparison of intensive and none surveillance for a 5-year survival resulted in an odds ratio of 2.606 (95\% CI 1.983-3.425) (Table 3).

Table 3. Relative Risk for 5-year survival after curative resection and for 3-year survival after diagnosis of recurrent disease.

$\begin{array}{ll}\text { Odds-ratio } & \begin{array}{l}\text { 95\% confidence } \\ \text { interval }\end{array}\end{array}$

\begin{tabular}{lll}
\hline 5-year survival & & \\
Intensive vs. minimal & 1.480 & $1.135-1.929$ \\
Intensive vs. none & 2.606 & $1.983-3.425$
\end{tabular}

3-year survival after

recurrence

$\begin{array}{lll}\text { Intensive vs. minimal } & 1.917 & 1.007-3.651 \\ \text { Intensive vs. none } & 2.434 & 1.088-5.448\end{array}$

\section{RECURRENCE}

The overall rate of recurrence for both local and distant growth of tumor was $25.3 \%(\mathrm{n}=371)$. The 5year survival rates for patients with a recurrent disease was 32\% (intensive), 13\% (minimal) and 19\% (none), respectively with statistically significant differences between the groups of; intensive and minimal $(p=0.034)$, intensive and none $(p<0.0001)$, but not between minimal and. none ( $p=0.614$ ) surveillance (Fig. 2).

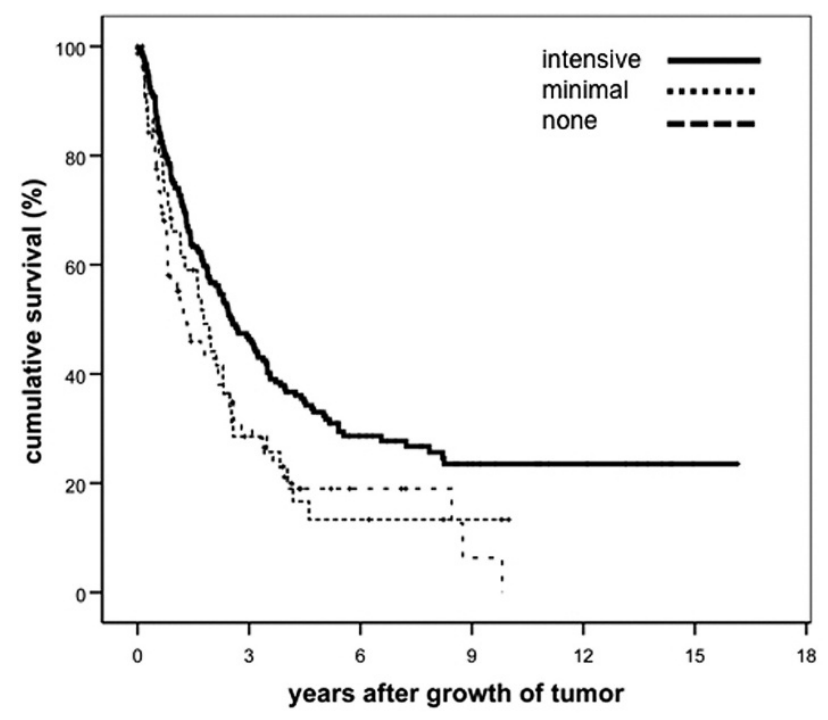

Fig. 2. Survival after recurrent disease depending on the intensity of surveillance prior to diagnosis.

The median time of survival after diagnosis of recurrent disease was 31 months (intensive), 21 months (minimal) and 16 months (none), respectively (intensive vs. minimal $\mathrm{p}<0.0001$, intensive vs. none $\mathrm{p}<0.0001$, minimal vs. none $\mathrm{p}<0.0001)$.

Patients who appeared asymptomatic at the time of diagnosis of recurrence differed among the groups. Out of the 243 patients with intensive surveillance 138 $(56.8 \%)$ did not show symptoms of recurrence in contrast to 18 out of 49 patients $(36.7 \%)$ with a minimal program $(p=0.27)$.

The odds ratio for a 3 -year survival comparing intensive and minimal surveillance was 1.917 (95\% CI 1.007-3.651). The comparison of intensive and none surveillance for a 3-year survival resulted in an odds ratio of 2.434 (95\%CI 1.088-5.448) (Table 3).

\section{LOCAL RECURRENCE}

Local recurrence occurred in 5.1\% $(\mathrm{n}=75)$ (Table 4). Recurrence of colon cancer occurred in $2.9 \%(n=23)$ and recurrence of rectal cancer in $7.7 \%(n=52)$. For both the rectum and the colon an $\mathrm{R} 0$ resection of re-

Table 4. Local Recurrence depending on site of primary tumor.

\begin{tabular}{lccc}
\hline Local recurrence & total & Colon & Rectum \\
\hline total & $75(5.2 \%)$ & $23(2.9 \%)$ & $52(7.7 \%)$ \\
extramural & $61(4.2 \%)$ & $20(2,5 \%)$ & $41(6.3 \%)$ \\
intramural & $14(9.7 \%)$ & $3(0,4 \%)$ & $11(1.7 \%)$ \\
\hline
\end{tabular}




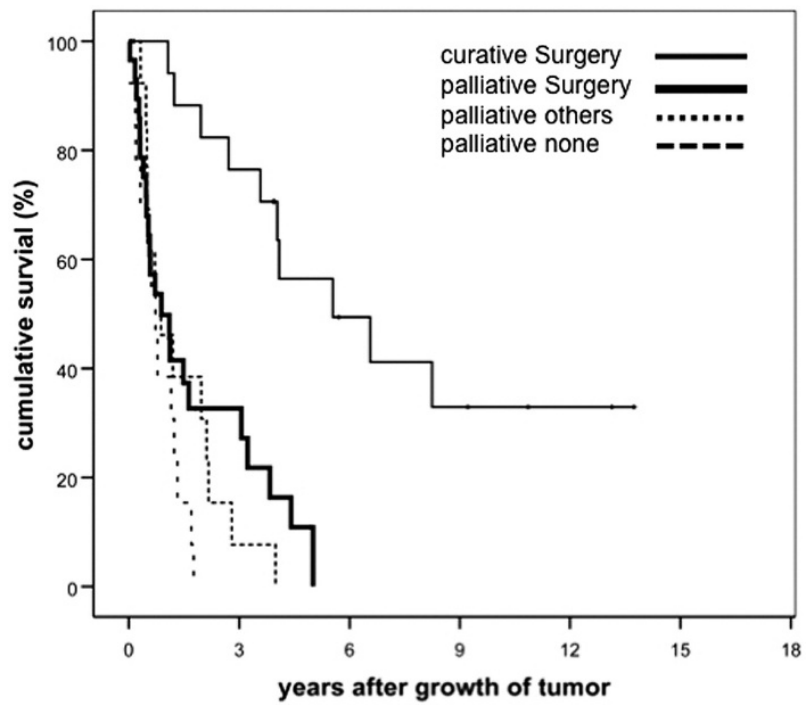

Fig. 3. Survival after local recurrence depending on the mode of therapy.

current disease was possible in $28 \%(14 / 50)$ for intensive, in $8 \%(1 / 13)$ for minimal and $25 \%$ (3/12) for none surveillance, but failed to show statistically significant differences (intensive vs. minimal $p=0.126$, intensive vs. none $p=0.238$, minimal vs. none $p=0.834$ ).

The 5-year survival rate for patients who had an R0 resection of recurrent disease was 57\% (Fig. 3). The 5year survival rate was $11 \%$ for those patients who received palliative surgical treatment and $0 \%$ for those patients receiving other palliative means (curative vs. palliative surgery $p<0.0001$, curative vs. other palliative means $p<0.0001)$. For the group who did not receive any treatment the 5-year survival rate was also $0 \%$ (curative therapy vs. no therapy $p<0.0001$ ). The median time of survival for the group with curative treatment was 67 months, for the group with palliative surgical therapy 11 months and in cases of other palliative or without treatment the median time of survival was11 and 8 months, respectively.

\section{Distant Metastases}

A total of 241 patients (16.4\%) presented with one or more metastases. An $\mathrm{R} 0$ resection with regard to the mode of surveillance was possible in 31.1\% (50/161) of patients with intensive, in $19.4 \%(7 / 36)$ with minimal and in 6.8\% (3/44) with none surveillance (intensive vs. minimal $p=0.165$; intensive vs. none $p=$ 0.002 , minimal vs. none $p=0.215$ ).

The liver was the most common site for distant recurrence with $8.4 \%$ (124) of all patients effected. Metastases of the lung occurred in $5.4 \%$ (79) of the total. In 7.6\% (112) of all patients metastases were localized elsewhere.

Isolated liver metastases were detected in 5.2\% (76) of patients and a curative resection could be performed in 40.8\% (31). The 5-year survival rate after curative surgery of isolated liver metastases was $47 \%$ (median time of survival 57 months) in contrast to a 5 -year survival rate of $0 \%$ (median time of survival 37 months) for palliative resection $(\mathrm{p}>0.05)$, palliative thermo ablation and palliative chemotherapy (median time of survival 20 months, curative vs. non-surgical palliative means $\mathrm{p}<0.0001)$. The 5 -year survival rate for isolated liver metastases without any therapy was also $0 \%$ (curative vs. none $\mathrm{p}<0.0001$ ).

In $2.8 \%$ (41) of patients the lung was affected solitarily. Here, a total of $46.4 \%(n=19)$ could be resected curatively. After curative resection of isolated metastases of the lung, the 5-year survival rate was $66 \%$, in contrast to a 5 -year survival rate of $0 \%$ (median time of survival 23 months) for palliative surgery (curative vs. palliative resection $p=0.125)$. Other palliative non-surgical means had a 5 -year survival rate of 14\% (median time of survival 17 months, curative vs. other palliative means $p=0.002$ ). The 5 -year survival rate for isolated lung metastases without any therapy was also $0 \%$ (curative vs. none $p<0.0001$ ).

\section{Discussion}

Since the introduction of surveillance after resection for colorectal cancer there has been a constant debate on how to practice follow-up [16-19]. Several studies have evaluated the effect of surveillance regarding outcome after curative resection. A significant survival benefit from intensive postoperative surveillance has been shown in four separate meta-analyses [8-11]. Figuerdo et al. and Renehan et al. analyzed six and five prospective, randomized trials, respectively, covering 1679 and 1342 patients [8, 10]. Renehan and colleagues stated that intensive follow up may improve survival because of earlier detection and treatment of recurrent disease. They added that it may also be associated with non-specific factors, such as a lesser psychological burden in patients undergoing surveillance. The detection rates in their meta-analysis for all local recurrences and hepatic metastases were similar to those quoted in the other studies. However, intensive follow-up was associated with a reduced time to first relapse and increased detection of isolated local recurrences [10].

Tjandra et al. presented a meta-analysis of eight randomized clinical trials with 2923 patients included. In this expanded systematic review they could confirm - consistent with five previous meta-analyses - a reduction of mortality and an improvement in curative reoperation rate with more intensive surveillance. Of the eight prospective trials, the largest study was the large-scale multicenter European study GILDA (Gruppo Italian di Lavoro per la Diagnosi Anticipata), which had enrolled a total of 985 patients. This trial was conducted to provide the first evidence, based on an adequately powered randomized trial to determine the optimal follow-up strategy for colorectal cancer patients. Recruitment in the US still continues and therefore, however, the duration of follow-up was relatively short at 14 months and data on longer-term surveillance limited $[11,20]$. In contrast to the results obtained in these meta-analyses, only two of the eight randomized studies suggested a significant improvement in survival for those undergoing intensive surveillance $[5,7,17,20-25]$.

The few retrospective studies available are very heterogenous regarding the size of the cohorts, the patients' characteristics and the time of follow-up [26- 
31]. Bergamaschi et al.'s cohort included 800 patients, which has been the largest retrospective study on this matter [26]. They reported a significantly higher rate of resectable recurrent disease and an improvement in survival after curative resection of recurrent disease in patients who underwent intensive follow-up. Castells et al. presented similar results in their study of $199 \mathrm{pa}-$ tients [27].

Another prospective, randomized study stated a significant difference in survival depending on the intensity of surveillance only for patients with stage II disease and rectal tumors [7]. The results of this randomized controlled trial suggest that, although no difference on overall survival was observed in the whole series, patients with colorectal cancer who have undergone curative resection may benefit from a more intensive surveillance strategy. This assumption was based on the fact that the intensive strategy increased the proportion of resectable tumor recurrences in the whole series as well as in some subsets of patients; more importantly, it increased the probability of overall survival in patients with stage II lesions or rectal tumors[7]. In contrast, Komborozos et al. could not reveal a difference in neither the rate of $\mathrm{R} 0$-resection rate for recurrent disease nor the overall rate of survival after surgical treatment of recurrence [29].

Our analysis of prospective, not randomized data supports the view that intensive surveillance implies a benefit in outcome. We were able to demonstrate a significant difference in overall survival with a benefit for patients who undergo intensive surveillance. The increasing difference in the 5 -year survival rate following primary surgery depending on the intensity of surveillance emphasizes the importance to encourage patients to participate in such programs. Our results are based on data retrieved from a follow-up, which in median was 70 months, a much longer period than that of most other studies. In addition, with 1469 patients included, we were able to analyze a cohort much larger than in other trials, both prospective and restrospective.

In the present analysis, the overall rate of local recurrence was significantly lower than the rate reported in 24 independent studies that were reviewed by Ohlsson and Pålsson [24]. We noted a rate of $5 \%$ in our collective compared to a median rate of $13 \%$ (range of 5 to $23 \%$ in this meta-analysis.

The results of our study show a significant improvement in survival for patients who underwent intensive surveillance prior to the diagnosis of a recurrent disease. Notably, only intensive surveillance improved survival for the group of patients with a recurrent tumor. Minimal surveillance had a similarly low effect on survival as none surveillance. In parallel, a meta-analysis by Renehan and colleagues revealed that intensive follow-up was associated with a significantly earlier detection of all recurrences. However, the rate of $\mathrm{R} 0$-resections for recurrent disease were not evaluated [10].

Other studies were able to show a significant improvement in $\mathrm{R} 0$ resection rates for the group that underwent intensive surveillance [7]. However, despite the higher rates of $\mathrm{R} 0$ resections - contrary to our results - the data of this multicenter, randomized trial did not show a benefit in survival for patients undergoing intensive surveillance prior to a recurrent disease [7].
The data presented suggest that the higher rate of $\mathrm{R} 0$ resections of a recurrent disease contributes to the significantly improved survival. However, the differences in $\mathrm{R} 0$ resection rate of local recurrence were not statistically significant comparing the groups with intensive, minimal and none surveillance. The reason for the lack of significance becomes obvious if one considers the small number of patients in the distinct groups, especially the one with none surveillance.

The survival after resection of a recurrent disease was significantly improved for the group with intensive surveillance. However, with regard to the lack of significance due to the small number of patients we cannot comment on a possible difference in R0-resection rates for recurrent disease and an according improvement of survival between the groups that underwent minimal and none surveillance. In contrast, the differences in $\mathrm{R} 0$ resection rates for distant metastases differed significantly and thus contributed to the improved survival.

Our results are based on surveillance according to the ASCO guidelines, where chest X-ray was recommended on a regular basis. The modifications of 2005 imply a CT-scan of the abdomen and the thorax to be performed annually in cases of colorectal cancer with a higher risk of recurrence [13]. Consequently, this might lead to a higher percentage of detected recurrences which are eligible for R0-resection. However, Rodriguez-Moranta et al. performed CT-scans on a regular basis and could demonstrate a benefit in survival for the group with intensive surveillance for stage II disease but not for stage III disease [7]. In addition, they were able to show a benefit for those with a primary tumor located in the rectum but not for those with a primary located in the colon. Generally, the use of CT-imaging in surveillance is performed irregularly among the different studies which makes a comparison somewhat difficult $[5,7,27]$.

The data presented should emphasize the importance to continue evaluations on the field of postoperative surveillance after primary resection for CRC. This includes the analysis of a definite improvement in survival due to a correctly adapted surveillance program and identification of patients who profit from another resection of a recurrent tumor and/or metastases. Furthermore, consideration of the psychological burden, which each surveillance appointment implies, and improvement in costs resulting from a correctly devised program remain important issues to be approached on this matter. Together with a modification of surgical techniques, adjuvant therapy and the introduction of other biochemical markers, it seems probable that given recommendations for surveillance will constantly have to be reviewed and modified.

\section{CONCLUSION}

The results of the study presented indicate a significantly improved survival for patients who undergo a systematic and intensified surveillance after curative resection of colorectal cancer. Although we need an ongoing debate on how to devise the ideal program of surveillance after curative resection for colorectal cancer we could show a benefit in overall survival and in 
survival after resection of recurrent disease for those patients who undergo intensive surveillance according to the ASCO guidelines.

Acknowledgements: Mrs. Claudia Killaitis is greatfully acknowledged for keeping the database updated.

\section{REFERENCES}

1. Jemal A, Siegel R, Ward E, Hao Y, Xu J, Murray T, Thun MJ: Cancer statistics, 2008. CA Cancer J Clin 2008;58:7196.

2. Boyle P, Ferlay J: Cancer incidence and mortality in Europe, 2004. Ann Oncol 2005;16:481-488.

3. Weitz J, Koch M, Debus J, Hohler T, Galle PR, Buchler MW: Colorectal cancer. Lancet 2005;365:153-165.

4. Bruinvels DJ, Stiggelbout AM, Kievit J, van Houwelingen HC, Habbema JD, van de Velde CJ: Follow-up of patients with colorectal cancer. A meta-analysis. Ann Surg 1994;219:174-182.

5. Pietra N, Sarli L, Costi R, Ouchemi C, Grattarola M, Peracchia A: Role of follow-up in management of local recurrences of colorectal cancer: a prospective, randomized study. Dis Colon Rectum 1998;41:1127-1133.

6. Quentmeier A, Schlag P, Smok M, Herfarth C: Re-operation for recurrent colorectal cancer: the importance of early diagnosis for resectability and survival. Eur J Surg Oncol 1990;16:319-325.

7. Rodriguez-Moranta F, Salo J, Arcusa A, Boadas J, Pinol V, Bessa X, Batiste-Alentorn E, Lacy AM, Delgado S, Maurel J, Pique JM, Castells A: Postoperative surveillance in patients with colorectal cancer who have undergone curative resection: a prospective, multicenter, randomized, controlled trial. J Clin Oncol 2006;24:386-393.

8. Figueredo A, Rumble RB, Maroun J, Earle CC, Cummings B, McLeod R, Zuraw L, Zwaal C: Follow-up of patients with curatively resected colorectal cancer: a practice guideline. BMC Cancer 2003;3:26.

9. Jeffery GM, Hickey BE, Hider P: Follow-up strategies for patients treated for non-metastatic colorectal cancer. Cochrane Database Syst Rev 2002:CD002200.

10. Renehan AG, Egger M, Saunders MP, O'Dwyer ST: Impact on survival of intensive follow up after curative resection for colorectal cancer: systematic review and metaanalysis of randomised trials. Bmj 2002;324:813.

11. Tjandra JJ, Chan MK: Follow-up after curative resection of colorectal cancer: a meta-analysis. Dis Colon Rectum 2007;50:1783-1799.

12. Benson AB, 3rd, Desch CE, Flynn PJ, Krause C, Loprinzi CL, Minsky BD, Petrelli NJ, Pfister DG, Smith TJ, Somerfield MR: 2000 update of American Society of Clinical Oncology colorectal cancer surveillance guidelines. J Clin Oncol 2000;18:3586-3588.

13. Desch CE, Benson AB, 3rd, Somerfield MR, Flynn PJ, Krause C, Loprinzi CL, Minsky BD, Pfister DG, Virgo KS, Petrelli NJ: Colorectal cancer surveillance: 2005 update of an American Society of Clinical Oncology practice guideline. J Clin Oncol 2005;23:8512-8519.

14. Oevermann E, Herold A, Schiedeck T, Bruch H: Followup for colorectal cancer. Coloproctolgy 1999;21:84-92.

15. Oevermann E, Herold A, Schiedeck T, Bruch H: Followup for colorectal cancer: Actual trends. Coloproctology 2005;27:280-288.

16. Johnson FE, Longo WE, Vernava AM, Wade TP, Coplin MA, Virgo KS: How tumor stage affects surgeons' surveillance strategies after colon cancer surgery. Cancer 1995; 76:1325-1329.

17. Secco GB, Fardelli R, Gianquinto D, Bonfante P, Baldi E, Ravera G, Derchi L, Ferraris R: Efficacy and cost of risk-adapted follow-up in patients after colorectal cancer surgery: a prospective, randomized and controlled trial. Eur J Surg Oncol 2002;28:418-423.

18. Vernava AM, 3rd, Longo WE, Virgo KS, Coplin MA, Wade TP, Johnson FE: Current follow-up strategies after resection of colon cancer. Results of a survey of members of the American Society of Colon and Rectal Surgeons. Dis Colon Rectum 1994;37:573-583.

19. Virgo KS, Wade TP, Longo WE, Coplin MA, Vernava AM, Johnson FE: Surveillance after curative colon cancer resection: practice patterns of surgical subspecialists. Ann Surg Oncol 1995;2:472-482.

20. Grossmann EM, Johnson FE, Virgo KS, Longo WE, Fossati R: Follow-up of colorectal cancer patients after resection with curative intent-the GILDA trial. Surg Oncol 2004;13:119-124.

21. Kjeldsen BJ, Kronborg O, Fenger C, Jorgensen OD: A prospective randomized study of follow-up after radical surgery for colorectal cancer. Br J Surg 1997;84:666-669.

22. Makela JT, Laitinen SO, Kairaluoma MI: Five-year follow-up after radical surgery for colorectal cancer. Results of a prospective randomized trial. Arch Surg 1995;130: 1062-1067.

23. Ohlsson B, Breland U, Ekberg H, Graffner H, Tranberg KG: Follow-up after curative surgery for colorectal carcinoma. Randomized comparison with no follow-up. Dis Colon Rectum 1995;38:619-626.

24. Ohlsson B, Palsson B: Follow-up after colorectal cancer surgery. Acta Oncol 2003;42:816-826.

25. Schoemaker D, Black R, Giles L, Toouli J: Yearly colonoscopy, liver CT, and chest radiography do not influence 5-year survival of colorectal cancer patients. Gastroenterology 1998;114:7-14.

26. Bergamaschi R, Arnaud JP: Routine compared with nonscheduled follow-up of patients with "curative" surgery for colorectal cancer. Ann Surg Oncol 1996;3:464-469.

27. Castells A, Bessa X, Daniels M, Ascaso C, Lacy AM, García-Valdecasas JC, Gargallo L, Novell F, Astudillo E, Filella X, JM P: Value of postoperative surveillance after radical surgery for colorectal cancer: results of a cohort study. Dis Colon Rectum 1998;41(6):714-723; discussion 723-714.

28. Eckardt VF, Stamm H, Kanzler G, Bernhard G: Improved survival after colorectal cancer in patients complying with a postoperative endoscopic surveillance program. Endoscopy 1994;26:523-527.

29. Komborozos VA, Skrekas GJ, Pissiotis CA: The contribution of follow-up programs in the reduction of mortality of rectal cancer recurrences. Dig Surg 2001;18:403-408.

30. Ovaska J, Jarvinen H, Kujari H, Perttila I, Mecklin JP: Follow-up of patients operated on for colorectal carcinoma. Am J Surg 1990;159:593-596.

31. Pugliese V, Aste H, Saccomanno S, Bruzzi P, Bonelli L, Santi L: Outcome of follow-up programs in patients previously resected for colorectal cancer. Tumori 1984;70: 203-208.

Received: July 22, 2009 / Accepted: August 14, 2009

Address for correspondence:

Dr. med. Tilman Laubert

Department of Surgery

University of Schleswig-Holstein

Campus Lübeck

Ratzeburger Allee 160

23538 Lübeck

Germany

Phone: +49-451-500 2001

Fax: $\quad+49-451-5002069$

e-mail: tilman.laubert@uk-sh.de 